\title{
Simulation of Brain Pulsation for Training Cerebrovascular Bypass Surgery Using Vibration Massage Seat
}

\author{
Sajjad Muhammad* and Ahmad Hafez \\ Department of Neurosurgery, University of Helsinki and Helsinki University Hospital, Finland
}

Received: 㠿: September 19, 2018; Published: 眥 September 27, 2018

*Corresponding author: Sajjad Muhammad, Helsinki University and Helsinki University Hospital, Helsinki, Finland

\section{Background}

Cerebral Revascularization procedure or bypass is procedure where a neurosurgeon uses existing vessels in the head and brain or vessels harvested from another part of the body to either replace blood flow in parts of the brain that are hypoperfused or to augment blood flow in portions of the brain that are under risk of ischemia and tissue damage. Cerebrovascular bypass is used to treat selected patients with decreased blood flow through their carotid arteries, patients with large aneurysms, patients with moyamoya disease as well as some patients with brain tumors. Due to very low number of such procedures, it is necessary to establish experimental training for bypass surgery to keep the practical skills intact for the neurosurgeons. Bypass training on vessels isolated from chicken wing is an excellent tool for training neurosurgeon, s skills. However, this experimental setting is static and do not simulate the brain pulsation movements.

\section{Description}

Here we established the experimental setting using vibrator massage seat under the prepared training vessels. The vessels were prepared from chicken wings and fixed on a head frame with a depth of $3 \mathrm{~cm}$. Head frame was placed on the vibrator massage seat. A setting with frequency 60 / minute nicely simulated brain pulsation similar to heart beat of 60 / min leading to similar brain pulsation. A 10-0 suture was used to perform the end to side anasthamosis under the exoscope (Karl Storz $\mathrm{GmH}$ ) using a magnification of 15 times at a focal distance of $200 \mathrm{~mm}$. Our video demonstration shows that suturing under static condition is much easier than in condition with simulated brain pulsation. It is more challenging and needs more concentration and better hand motor skills and hand- eye coordination to perform sutures.

\section{Conclusion}

Our technical development is easy and simple for a better training of bypass surgery for young neurosurgeons.

\section{Acknowledgement}

We are thankful to Ehrnrooth Foundation for the Funding to SM for a clinical vascular and skull base fellowship at the department of Neurosurgery.

Video 1 
ISSN: 2574-1241

DOI: 10.26717/BJSTR.2018.09.001799

Sajjad Muhammad. Biomed J Sci \& Tech Res

(c) (i) This work is licensed under Creative

Submission Link: https://biomedres.us/submit-manuscript.php

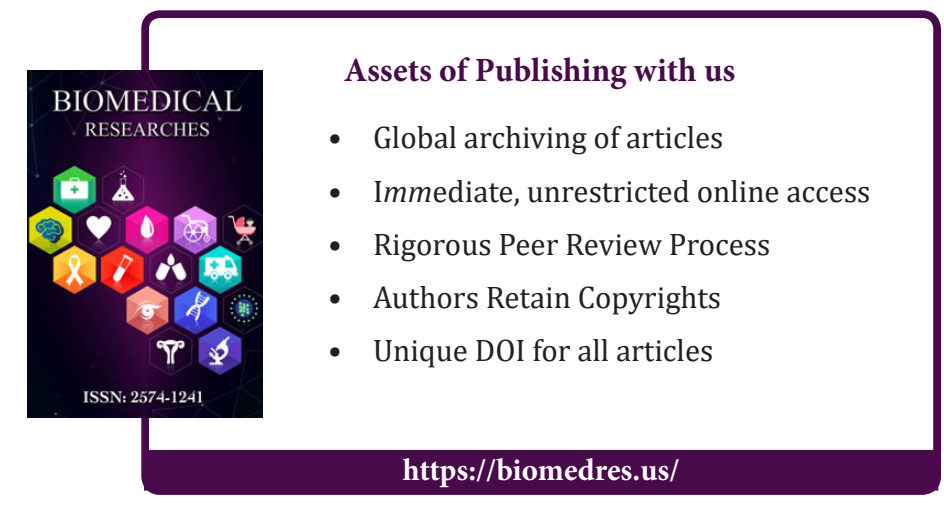

